\title{
Mental Imagery for Full and Upper Human Bodies: Common Right Hemisphere Activations and Distinct Extrastriate Activations
}

\author{
Olaf Blanke · Silvio Ionta • Eleonora Fornari • \\ Christine Mohr $\cdot$ Philippe Maeder
}

Received: 3 November 2009/ Accepted: 12 February 2010

(C) Springer Science+Business Media, LLC 2010

\begin{abstract}
The processing of human bodies is important in social life and for the recognition of another person's actions, moods, and intentions. Recent neuroimaging studies on mental imagery of human body parts suggest that the left hemisphere is dominant in body processing. However, studies on mental imagery of full human bodies reported stronger right hemisphere or bilateral activations. Here, we measured functional magnetic resonance imaging during mental imagery of bilateral partial (upper) and full bodies. Results show that, independently of whether a full or upper body is processed, the right hemisphere (temporoparietal cortex, anterior parietal cortex, premotor cortex, bilateral superior parietal cortex) is mainly involved in mental imagery of full or partial human bodies. However, distinct activations were found in extrastriate cortex for
\end{abstract}

O. Blanke $(\bowtie) \cdot$ S. Ionta

Laboratory of Cognitive Neuroscience, Brain-Mind Institute, Ecole Polytechnique Fédérale de Lausanne (EPFL), Station 19, 1015 Lausanne, Switzerland

e-mail: olaf.blanke@epfl.ch

O. Blanke $\cdot$ C. Mohr

Department of Neurology, University Hospital, Geneva, Switzerland

E. Fornari

Department of Radiology, CIBM-CHUV Unit, Centre

Hospitalier Universitaire Vaudois and University of Lausanne,

Lausanne, Switzerland

C. Mohr

Department of Experimental Psychology, University of Bristol,

Bristol, UK

P. Maeder

Department of Radiology, Centre Hospitalier Universitaire

Vaudois and University of Lausanne, Lausanne, Switzerland partial bodies (right fusiform face area) and full bodies (left extrastriate body area). We propose that a common brain network, mainly on the right side, is involved in the mental imagery of human bodies, while two distinct brain areas in extrastriate cortex code for mental imagery of full and upper bodies.

Keywords Mental rotation - Body - Neuroimaging · Out-of-body $\cdot$ Neuropsychology $\cdot$ fMRI

\section{Introduction}

Human bodies provide a particularly rich source of visual social information. Behavioural, neuropsychological, and neuroimaging studies suggest that processing human bodily stimuli involves brain regions that are at least partially different from those sub-serving the processing of noncorporeal objects (Ionta et al. 2010; Wraga et al. 2005; Creem et al. 2001; Bonda et al. 1995; Parsons 1987a, b). With respect to neural mechanisms, neuropsychological findings suggest that the left hemisphere might be dominant for the processing of body parts (Schwoebel and Coslett 2005; Guariglia et al. 2002; Sirigu et al. 1991; Ogden 1985), although own body illusions and deficits in corporeal awareness have been linked primarily to the right hemisphere (Blanke and Mohr 2005; Berlucchi and Aglioti 1997). Investigating the differences between different modalities of mental imagery (i.e., motor vs. visual), it has been suggested that left brain regions are more involved in motor imagery, whereas right brain regions are more involved in visual imagery (Sirigu and Duhamel 2001; Tomasino and Rumiati 2004). This has been extended by neuroimaging work studying mental imagery in healthy subjects and revealing a left-hemisphere dominance in 
body processing, showing the activation of superior parietal lobule (SPL) and cortex at the intraparietal sulcus (IPS) during mental imagery for human body parts (Overney and Blanke 2008; Overney et al. 2005; de Jong et al. 2001; Bonda et al. 1995). Others reported bilateral parietal activations (Kosslyn et al. 1998; Parsons et al. 1995). Activations at the temporo-parietal junction (TPJ) are generally bilateral or have been found mainly in the right (Blanke et al. 2005) or the left hemisphere (Zacks et al. 1999). Finally, the majority of the studies that employed stimuli depicting human body parts such as hands or arms reported stronger left parietal activations (Overney and Blanke 2008; Overney et al. 2005; de Jong et al. 2001; Bonda et al. 1995), whereas studies depicting full bodies revealed more bilateral (Zacks et al. 1999, 2002) or right parietal and/or temporo-parietal activations (Blanke et al. 2005).

Given the important roles played by neural body representations in mental imagery in the processing of human bodies, we investigated the neural differences during mental imagery of partial (upper) bodies and full bodies using fMRI. Two main reasons guided this choice. First, body stimuli in previous studies were generally presented as a single left or right arm or hand, whereas studies on mental imagery of full human bodies used bilateral body stimuli. Using bilateral stimuli of the upper part of the human body (Reed et al. 2006) would allow comparison with mental imagery of bilateral stimuli of the full body. Second, our study was motivated by differences in neural coding for upper and full human bodies as suggested by clinical evidence from neurological patients with illusory own body perceptions such as autoscopic hallucinations and out-of-body experiences (Blanke et al. 2005; Blanke and Metzinger 2009). During both illusions patients experience seeing a second own body in extrapersonal space. During out-of-body experiences the illusory body is usually perceived as a full body, whereas during autoscopic hallucination it is mostly the upper part of the body that is perceived (Brugger 2002; Blanke and Mohr 2005). We therefore adapted a classical mental imagery task (Parsons 1987a) that had already been employed in electrical neuroimaging work (Blanke et al. 2005; Zacks et al. 1999) and designed the upper body based on patients with autoscopic allucinations.

\section{Materials and Methods}

\section{Subjects}

Fourteen healthy volunteers (7 male; mean age $28 \pm$ 2.9 years) participated in the study. Handedness was evaluated using the Oldfield-Edinburgh questionnaire (Oldfield 1971). Thirteen participants were right-handed (scores between +0.8 and +1 ) and one participant was left handed (score of -0.9). All participants had normal or corrected-to-normal vision, and no history of neurological or psychiatric disorders as indicated by a self-report. Participants gave written informed consent prior to inclusion in the study, which have been approved by the Ethical Committee of the University Hospital of Lausanne (Switzerland). The procedure was performed in accordance with the ethical standards laid down in the Declaration of Helsinki 1964.

\section{Stimuli}

Stimuli were modified from stimuli previously used in mental own body transformation tasks (OBT-task) by others (Parsons 1987a; Zacks et al. 1999) as well as our group (Blanke et al. 2005; Mohr et al. 2006). Stimuli consisted of schematic human figures that could be facing toward or away from the participant (Fig. 1). Front- and back-facing figures had the same outline and differed only in the rendering of the figure's clothing and the presence of a face or of the back of a head (Fig. 1a). The figure's hands were marked such that one hand appeared to be wearing a grey glove and a black ring at the wrist. This indication of side could appear on the right or on the left hand. The task performed with such stimuli was called OBT-task for full bodies $\left(\mathrm{OBT}_{\mathrm{f}}\right.$-task). In addition participants performed the OBT-task with other visual stimuli, taken from the same schematic human figure but consisting only of the upper part of the body. Again, front- and back-facing figures had the same outline and differed in the rendering of the figure's clothing and the presence of a face or of the back of a head (Fig. 1b). The figure's ears were marked such that one ear was grey and had a black earring. This indication of side could appear on the right or on the left ear. The

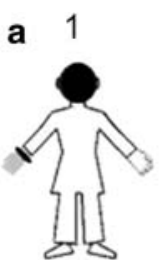

left

b

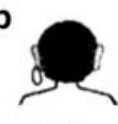

left

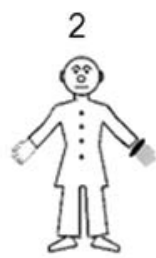

left

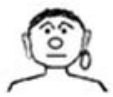

left

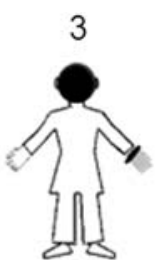

right

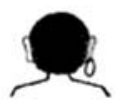

right

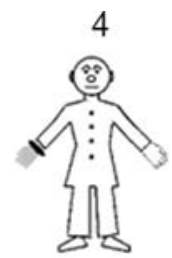

right

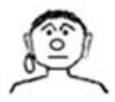

right
Fig. 1 Stimuli. The eight stimuli used in both the OBT-task and the LAT-task. Within rows $\mathbf{a}$ and $\mathbf{b}$, the first and the third figure are examples of back-facing stimuli, the second and the fourth figure are examples of front-facing stimuli. Correct responses in the OBT-task are indicated below each figure 
OBT-task with this kind of stimuli was called OBT-task for upper bodies $\left(\mathrm{OBT}_{\mathrm{u}}\right.$-task). The presentation duration and size of stimulus, as well as the interstimulus interval were identical to the $\mathrm{OBT}_{\mathrm{f}}$-task. Full and partial body stimuli were controlled for overall luminance.

Stimuli appeared for $1000 \mathrm{~ms}$ in the center of the computer screen $\left( \pm 6.0^{\circ} \times \pm 6.0^{\circ}\right.$ of visual angle $)$. An LCD projector with a refresh rate of $75 \mathrm{~Hz}$ displayed the stimuli. The projector was equipped with a photographic zoom lens projecting images onto a translucent screen in a custommade mirror box positioned inside the magnet. The mirror box was designed to minimize light reflection. The interstimulus interval was $1000 \mathrm{~ms}$ (equal to stimulus duration). The choice of the body part to mark in each type of stimulus (hand for full bodies and ear for upper bodies) was chosen in order to keep the target across stimuli as similar as possible in size and in position within the visual fields across conditions.

\section{Procedure}

In both tasks, participants were asked to make right-left judgements of the schematic full or upper human figure after having imagined themselves to be in the figure's body position and visuo-spatial perspective. In the $\mathrm{OBT}_{\mathrm{f}}$-task, participants were instructed to indicate whether the marked hand of the figure would be their right or left hand. In the $\mathrm{OBT}_{\mathrm{u}}$-task, participants were instructed to indicate whether the marked ear of the figure would be their right or left ear. In both tasks participants were instructed to respond with a button press on a serial response box as fast and accurate as possible and to always perform the mental transformation of their body prior to giving the response. At the end of the experiment participants were asked to report if they could perform the OBT task as suggested or if they used another strategy. Responses were given with the right hand. Left judgements were indicated by a button press of the index finger and right judgements by a button press of the middle finger. Participants were previously trained on the task on a computer and were asked to answer with their preferred hand on a keyboard. All the participants chose to answer with the right hand. This strategy was accepted because it has been shown that there is a left hand advantage in behavioural data during self-recognition task (Keenan et al. 1999) and we were concerned not to bias behavioural results towards one condition. Moreover, since participants were scanned during one single session, it was not possible to counter-balance hands within participants across sessions.

In two control conditions (lateralization task or LATtask), the same visual stimuli (full bodies, upper bodies) were used, but participants decided whether the indicated hand or ear was on the right or the left side of the computer screen (no imagined change in own body position and visuo-spatial perspective). These two conditions $\left(\mathrm{LAT}_{\mathrm{f}}\right.$ task; $\mathrm{LAT}_{\mathrm{u}}$-task) were carried out in order to dissociate central mechanisms of OBT from those due to the mere perception of the human body and right-left judgements. Responses were also given with the right hand. Again, left judgements were indicated by a button press of the index finger and right judgements by a button press of the middle finger.

All participants performed a training session before being scanned. Blocks of 20 presentations of randomized images of different stimuli (upper bodies or full bodies) and different tasks (LAT-task or OBT-task) were alternated with $16 \mathrm{~s}$ of resting state during which only a fixation point was displayed. Each cycle described was repeated four times.

\section{Analysis of Behavioural Data}

Repeated measures ANOVAs were performed on the mean reaction times for correct responses with Task (OBT- vs. LAT-task), Body (Upper body vs. full body), and Orientation (back-facing vs. front-facing) as main factors. Posthoc comparisons were carried out using LSD tests.

\section{Recording and Analysis of fMRI Data}

BOLD fMRI acquisitions were performed with a head coil on a $1.5 \mathrm{~T}$ Siemens Magnetom Vision system equipped for echoplanar imaging. The participant's head was cushioned in the coil with a vacuum beanbag to prevent motion. Functional MRI images were acquired with an EPI gradient echo T2*-weighted sequence (FA 90, TE 66, pixel size $3.75 \times 3.75 \mathrm{~mm}$, acquisition time $1.7 \mathrm{~s}$, 16 slices of $5 \mathrm{~mm}$ with a gap of $1 \mathrm{~mm}$ ) with a TR $=4 \mathrm{~s}$ for a total of 20 acquisitions for each stimulus. fMRI pre-processing steps, conducted with SPM2 (Wellcome Department of Cognitive Neurology, London, UK), included realignment of intrasession acquisitions to correct for head movement, normalization to a standard template (Montreal Neurological Institute template, MNI) to minimize inter-participant morphological variability, and convolution with an isotropic Gaussian kernel (FWHM $=9 \mathrm{~mm}$ ) to increase signalto-noise ratio. Single participant analysis was performed according to the General Linear Model. The signal drift across acquisitions was removed with high-pass filter and global signal changes by proportional scaling. Statistical parametrical maps of the contrasts of interest were computed for each subject as input values for the group statistics based on Random Field Theory. In particular, the inferential statistics included a repeated measures ANOVA, and T-tests (Knyazeva et al. 2006; Klöppel et al. 2007; Henson et al. 2004). Only voxels with the height threshold set at $P<0.01(F>5.19)$ were considered 
significant in the $F$-tests performed to obtain ANOVA results. Post-hoc comparisons were then tested with the corresponding paired $T$-tests between conditions, thresholded for peak height at $P<0.001(T>3.85)$, in order to determine which specific comparison supported the effect. In each analysis, the extent threshold $k>30$ contiguous voxels, larger than the minimum number of voxels expected per cluster (Friston et al. 2003), were applied to SPMs. Corrections for multiple comparisons were used at a cluster level $[P$ (corrected) $<0.05]$. From the clusters showing a significant $P$ (corrected) value both in an ANOVA effect and in post-hoc paired $T$-test, we then extracted the mean percent signal change and variance for each condition in order to show the direction of the effect and the contribution of each separate condition. A sagittal T1-weighted 3D gradient-echo sequence (MPRAGE), 128 slices (with voxel size of $1 \times 1 \times 1.25 \mathrm{~mm}$ ), was also acquired as structural basis for brain segmentation and surface reconstruction.

\section{Results}

Behaviour

Statistical analysis revealed a significant main effect of Task $[F(1,13)=3464.30 ; P<0.001 ;$ Fig. 2$]$ with longer RTs observed in the OBT-task $(632.1 \pm 146.6 \mathrm{~ms})$ than the LAT-task (408.9 $\pm 101.6 \mathrm{~ms}$; Zacks et al. 1999; Blanke et al. 2005). The main effect of Orientation $[F(1,13)=21.12, P<0.001])$ revealed that reaction times were longer for front-facing figures $(526.6 \pm 160.0 \mathrm{~ms})$ than for back-facing figures $(509.2 \pm 158.2 \mathrm{~ms})$. The Task by Orientation interaction was also significant $[F(1,13)=4.69 ; P<0.03]$. Post-hoc analysis revealed that the OBT-task took longer than the LAT-task if the figure was seen as front-facing $(645.0 \pm 146.9 \mathrm{~ms})$ as compared to back-facing $(619.3 \pm 146.3 \mathrm{~ms})$. This front-back difference was specific for the OBT-task (for the LAT-task, front-facing figures: $412.8 \pm 112.1 \mathrm{~ms}$, back-facing figures: $408.3 \pm$ $88.8 \mathrm{~ms}$ ). Post-hoc analysis showed the significant difference between responses for front-facing and back-facing stimuli for the OBT-task $(P<0.001)$, but not for the LATtask $(P>0.05)$. There was no significant main effect of Body on reaction times [full bodies: $521.1 \pm 162.8 \mathrm{~ms}$; upper bodies: $515.3 \pm 168.1 \mathrm{~ms} ; F(1,13)=2.46, P=$ $0.12]$. With respect to error rates, statistical analysis revealed a significant main effect of Task $[F(1,13)=16.9$, $P<0.001]$ accounted for by the better performance with the LAT-task (99\%) with respect to the OBT-task $(95 \%)$, but not for Body or Orientation or any interaction (all $P>0.3$ ).
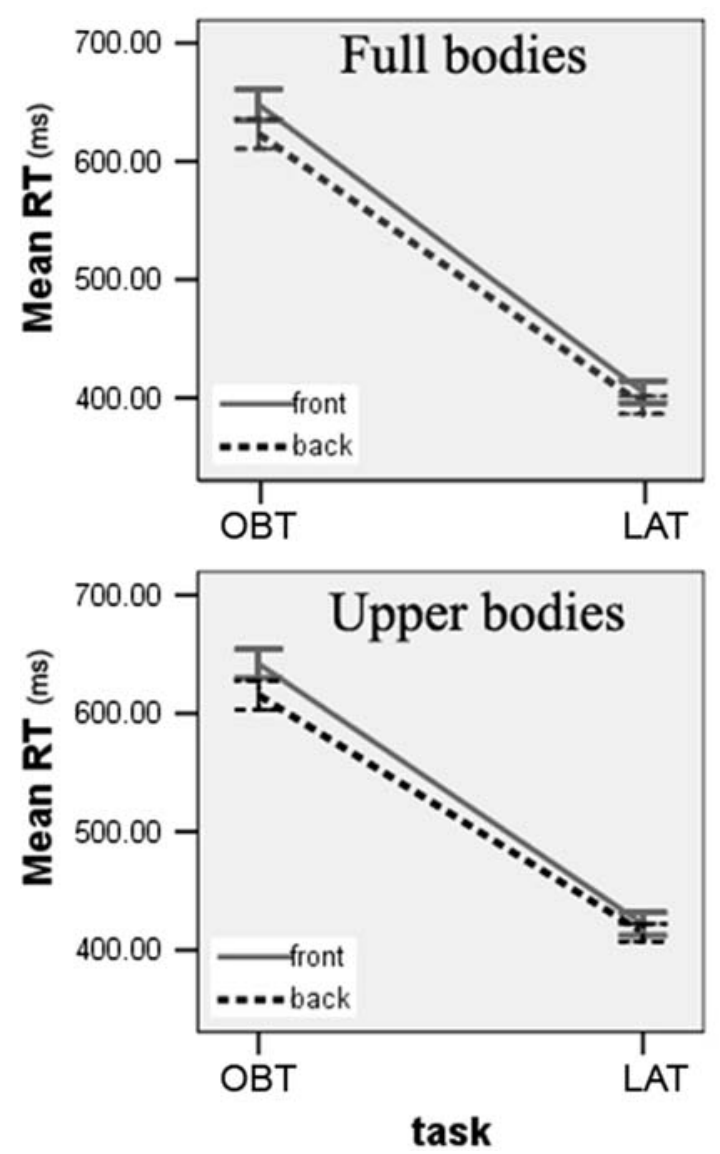

Fig. 2 Behavioural data. Behavioural data during OBT-task and lateralization task for full bodies and upper bodies. Solid lines represent the reaction time for front-facing figures while dotted lines for back-facing. Plots show the reaction time mean values \pm 2 standard errors

A general issue regarding mental imagery tasks is to control whether participants really perform the requested task. We think that our participants performed mental imagery as requested for several reasons. First, participants were repeatedly instructed to respond as fast and precisely as possible, but to always perform the mental transformation of their body prior to giving the response. Second, at the end of the experiment all subjects were asked to report how they performed the OBT task and whether this was as instructed. Four subjects who reported an alternative strategy (i.e., inversion of the response for the front-facing figures or rotation of the visually presented body around the $\mathrm{z}$-axes) were excluded from the study. Third, our behavioural results showed significant differences between responses to front-facing and back-facing stimuli in the OBT-task. This differed from the LAT-task in which such differences were not found. 


\section{fMRI}

First we will describe the brain regions showing a taskrelated effect independent from the stimulus type, then those regions showing a stimulus-related effect and finally those regions showing an interaction between the factors task and stimulus. Each described region was tested with the corresponding $T$-test supporting the effect and the mean value of percent signal change within the cluster and its variance for each condition were extracted and displayed as plot bars.

\section{Main Effect of Task}

The BOLD signal differed significantly in several brain regions when comparing OBT-task with LAT-task regardless of the stimulus type (Fig. 3a).

At the right TPJ a significant cluster was localized at the junction of the posterior superior temporal gyrus with the angular gyrus $(x, y, z=48,-42,18$; Fig. 3b). Post-hoc comparisons showed that both OBTf versus LATf $(T=4.52)$ and OBTu versus LATu $(T=4.05)$ comparison supported the effect although with different strength. Also the right dorsal premotor cortex $(x, y, z=51,12,21$; Fig. 3c) responded more strongly in the OBT-task than in the LAT-task for both stimuli (full bodies, $T=6.31$; upper bodies, $T=7.02$ ). No similar activations were found in the left premotor cortex. The BOLD response in parietal cortex showed two distinct locations of activation. The posterior peak was in the superior parietal lobule and found in both hemispheres $[x, y, z$ (left) $=-9,-78,48 ; x, y, z$ (right) $=9,-75,51$; Fig. 3d]. Both clusters were supported by OBTf versus LATf $[T($ left $)=7.55, T$ (right $)=$ 5.97] and OBTu versus LATu $[T($ left $)=4.65, T$ (right $)=$ 6.02] contrasts. The anterior peak was in the middle/anterior part of the intraparietal sulcus $[x, y, z$ (right) $=42$, $-48,39 ; x, y, z$ (left) $=-48,-45,36$; Fig. 3e]. Although this region also appeared bilaterally, its increase of
Fig. 3 fMRI Data: Main effect of task. Surface 3D display of the comparison between OBT and LAT-task regardless of whether full or upper bodies were shown. Color bar represents $F$ statistical values (a). For four regions the BOLD response is shown for the four experimental conditions (OBTf, LATf, OBTu, LATu) separately and expressed in mean percent of signal change \pm standard deviation for significant clusters. These areas are: right temporo-parietal cortex (b), right premotor cortex (c), right and left superior parietal lobule (d), and right and left intraparietal sulcus area (e). The location of each cluster of interest is shown in a representative slice, centered in its center of gravity. Both in the cluster display and in the plotbars, red refers to clusters in the right hemisphere, blue, in the left
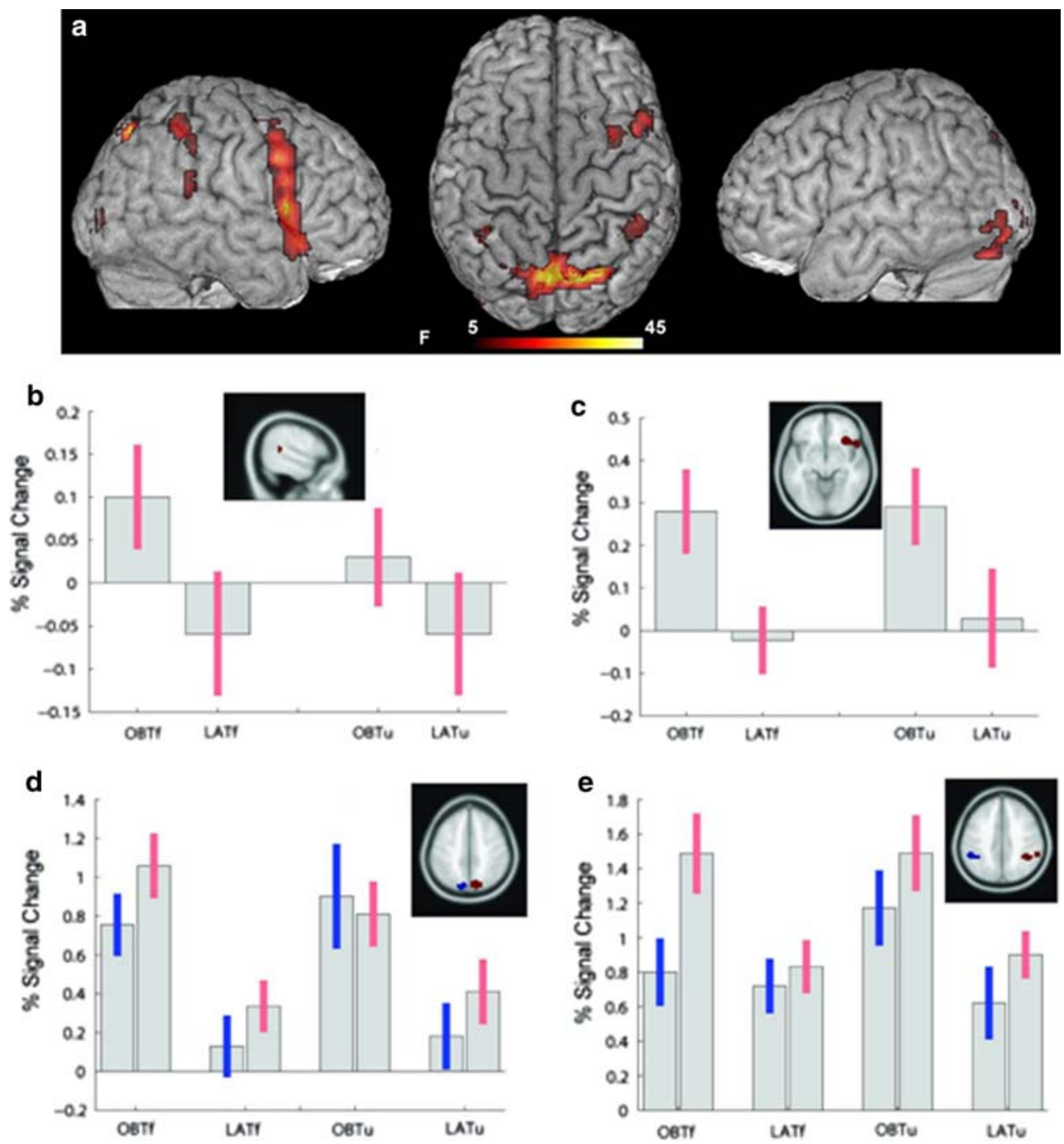
activation during the OBT task was supported by both $T$-contrasts only in the right hemisphere (OBTf vs. LATf, $T=7.28$ and OBTu vs. LATu, $T=6.08$ ). The cluster in the left hemisphere was characteristic mainly of the OBTu versus LATu contrast ( $T=4.98)$.

As can be noticed in Fig. 3, all described regions are characterized by an increase of activation during the OBTtask as compared to the LAT-task. There were no regions showing the opposite trend. Clusters present in extrastriate visual cortex were not supported by $T$-tests.

\section{Main Effect of Body and Interactions}

A main effect of body was found in one region with a significant activation in right fusiform cortex $(x, y, z=16$, $-80,-14$, Fig. 4a). A significant difference in BOLD response was found during the $\mathrm{OBT}_{\mathrm{u}}$-task relative to the activation during the $\mathrm{OBT}_{\mathrm{f}}$-task $(T=4.76)$. The same difference in the BOLD mean value was found when comparing $\mathrm{LAT}_{\mathrm{u}}$-task with $\mathrm{LAT}_{\mathrm{f}}$-task $(T=4.32)$. No similar activations were found at left FFA. Due to enhanced activation for the upper body stimuli containing large faces we argue that this activations reflects activity in FFA.

The interaction contrast showed an area in left extrastriate cortex, probably corresponding to the left EBA (Downing et al. 2001; Grossman and Blake 2002), differently activated across tasks and stimulus type. Left EBA $(x, y, z=-45,-83,-4 ;$ Fig. 4b) responded more strongly in the $\mathrm{OBT}_{\mathrm{f}}$-task compared to the $\mathrm{LAT}_{\mathrm{f}}$-task $(T=4.87)$ than in the $\mathrm{OBT}_{\mathrm{u}}$-task compared to the $\mathrm{LAT}_{\mathrm{u}}$-task (where the difference is not significant). No similar activation was found at the right EBA.

\section{Discussion}

The present results suggest that a mainly right hemispheric network of brain areas-including right TPJ, right FFA, right anterior parietal cortex, right premotor cortex, bilateral superior parietal cortex, and left EBA-is involved in mental imagery for human bodies. Whereas the right FFA
Fig. 4 fMRI data: Main effect of Body and interactions. Surface 3D display of the comparison between tasks depicting upper bodies and full bodies regardless of whether OBT- or LAT-task was performed. Right fusiform face area (FFA) showed main effect of Body (a). An area in left extrastriate cortex, probably corresponding to the extrastriate body area showed a

Task $\times$ Body interaction $(\mathbf{b}$; see text). Color bar represents $F$ statistical values. BOLD responses are shown for the four experimental conditions (OBTf, LATf, OBTu, LATu) separately and expressed in mean percent of signal change \pm standard deviation for significant clusters. The location of each cluster of interest is shown in a representative slice, centered in its center of gravity. Both in the cluster display and in the plotbars, red refers to clusters in the right hemisphere, blue, in the left
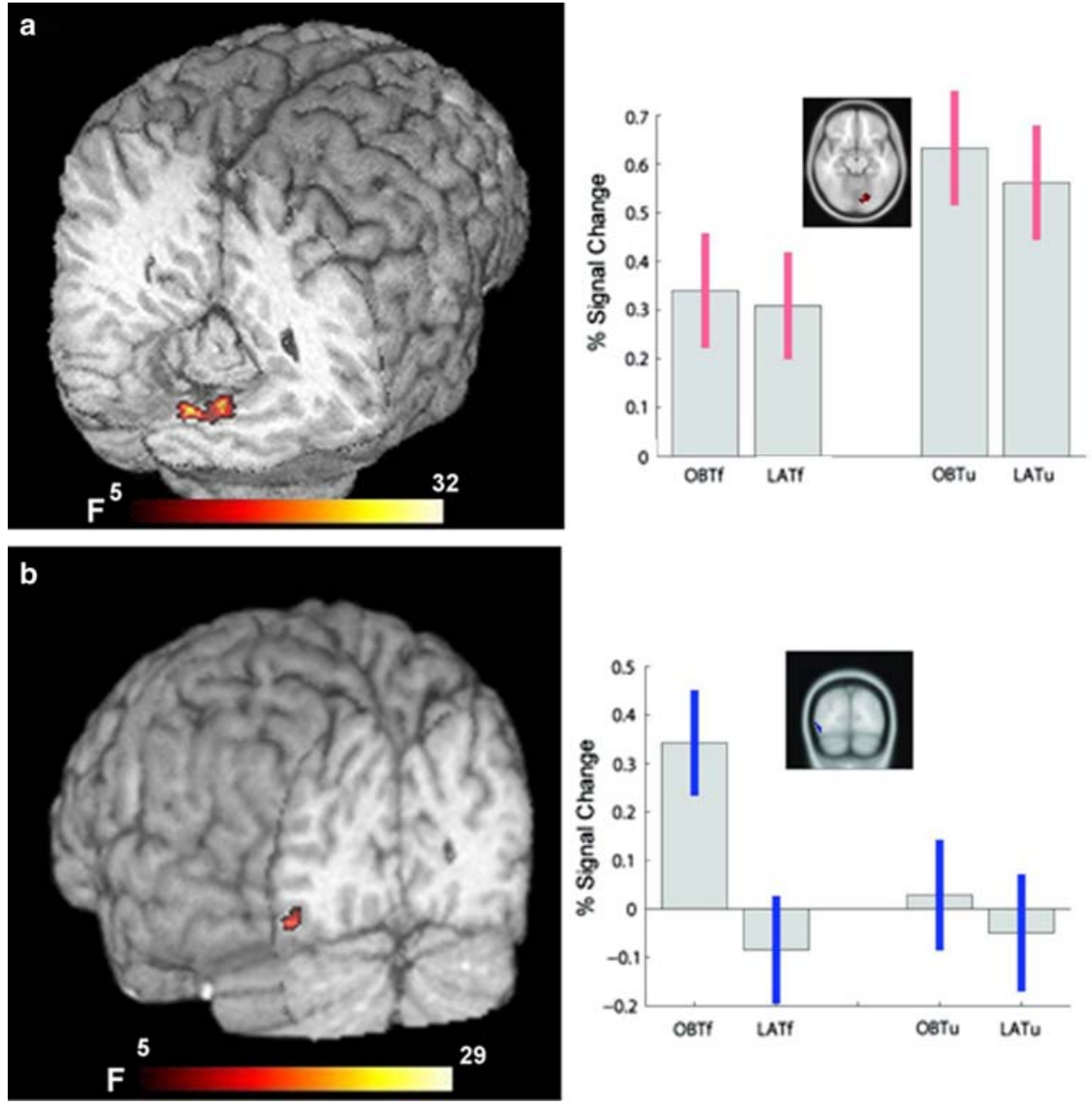
was activated during the presentation of upper bodies independent of the task, the left EBA coded for mental transformation only when full bodies were employed. These data corroborate and extend behavioural data (Reed et al. 2006) and clinical data (Blanke et al. 2004; Blanke and Mohr 2005) suggesting the presence of similar and distinct mechanisms in the processing of two important social stimuli-full and upper human bodies.

\section{Temporo-Parietal Junction}

Using evoked potential mapping and transcranial magnetic stimulation we have recently shown (Blanke et al. 2005) that the right TPJ is a key area for mental imagery of full human bodies but not for non-corporeal objects. The present fMRI results extend these latter findings showing that the junction of the right angular gyrus and posterior STG is also activated during mental imagery for bilateral upper bodies. The right angular gyrus has been previously shown to be involved in the perception of the body and self and in coding for egocentric visuo-spatial perspective taking (Vallar et al. 1999; Maguire et al. 1998), agency (Farrer and Frith 2002; Farrer et al. 2003; Chaminade and Decety 2002), and self-other distinction (Farrer and Frith 2002; Farrer et al. 2003; Ruby and Decety 2001, 2003, 2004; Chaminade and Decety 2002). In addition, the vestibular cortex is localized at the TPJ including the angular gyrus (Lobel et al. 1999; Fasold et al. 2002). The posterior STG and superior temporal sulcus area have also been shown to be crucial for several aspects of bodily processing by coding for various visual stimuli of human bodies such as in visual biological motion perception (Howard et al. 1996; Vaina et al. 2001; Grossman and Blake 2002), the visual perception of static human body parts including eyes, hands, mouths (Puce et al. 1998; Allison et al. 2000; Grossman and Blake 2002), as well as sensorimotor integration (Leube et al. 2003). The present data thus suggest that the right TPJ codes for mental own body transformations and imagined visuo-spatial perspective changes.

\section{Parietal Cortex}

The present data confirm that the parietal cortex is involved in mental imagery of full and upper bodies. Both OBTtasks led to strong bilateral activation of parietal cortex. This is concordant with activations at this site observed in most studies investigating the neural correlates of mental imagery of bodily stimuli (Bonda et al. 1995; Kosslyn et al. 1998; Zacks et al. 1999; Creem et al. 2001; de Jong et al. 2001; Lenggenhager et al. 2006), but also non-corporeal objects (Pegna et al. 1997; Harris and Miniussi 2003; Vingerhoets et al. 2001; Alivisatos and Petrides 1997; Carpenter et al. 1999; Kawamichi et al. 2007).
Whereas some earlier studies showed that mental imagery of body parts (such as arms and hands) predominantly activates left parietal cortex (Bonda et al. 1995; Kosslyn et al. 1998; Zacks et al. 1999; Creem et al. 2001; de Jong et al. 2001) we found bilateral or bilateral and right predominant parietal activations for both types of body stimuli that were used in the present study. We suggest that mental imagery of symmetrical body stimuli (such as full or upper bodies used here) activate parietal cortex bilaterally whereas mental imagery of asymmetrical or lateralized body parts more strongly relies on left parietal cortex. The fact that strong bilateral parietal activations were observed in both present tasks as well as in many previous studies using symmetrical non-corporeal objects (Pegna et al. 1997; Harris and Miniussi 2003; Vingerhoets et al. 2001; Alivisatos and Petrides 1997; Carpenter et al. 1999) would also suggest that bilateral parietal cortex is activated independent of the type of stimulus that is used for mental imagery when the stimulus is symmetrical. However further experiments directly comparing different partial body stimuli are necessary, especially as comparison across studies is difficult because different paradigms, such as two-stimuli or single-stimulus presentations as well as different imagery instruction have been applied, likely affecting the observed brain activation patterns (Vingerhoets et al. 2001).

Posterior parietal cortex is also a key region for the processing of spatial information with respect to egocentric and allocentric reference frames (Burgess 2008; Zacks et al. 2003; Cohen and Andersen 2002; Colby and Duhamel 1996). Whereas in an egocentric reference frame objects are represented relative to the subject, in an allocentric reference frames objects are represented relative to the environment (Volcic and Kappers 2008; Wraga et al. 1999). The current tasks involved both referential frames differently, with OBT loading on egocentric transformations and LAT on allocentric transformations. Enhanced activation of parietal cortex found here for the OBT-task with respect to the LAT-task might therefore be also related to differences in the spatial reference frame.

Given that the BOLD signal showed an increase during the OBT-tasks with respect to the LAT-tasks (Fig. 3) and that response times were faster for the LAT-task than for the OBT-tasks, it could be also argued that the observed main effect of task (as found in behavioural and fMRI results) is due to a difference in task difficulty (OBT vs. LAT). However, the behavioural results showing significant differences between response times to front-facing and back-facing stimuli in the OBT-task, but not the LAT-task, suggest that mental own body transformations significantly influenced task performance. Moreover, the fact that activations in some regions were accounting for both the Task (OBT vs. LAT) and Orientation (Back vs. Front) effects, 
and that these effects were interacting, suggest that these activities depended not only on task difficulty.

\section{Premotor Cortex}

Premotor cortex was activated during mental imagery independently of stimulus type, concordant with many previous studies using body parts and full bodies (Bonda et al. 1995; Kosslyn et al. 1998; Zacks et al. 1999; Creem et al. 2001). In the present study, activations in premotor cortex during both tasks were restricted to the right hemisphere. This right lateralization cannot be explained by response generation as responses were given with the right hand for all tasks and should have accordingly led to more prominent left premotor activation (Aziz-Zadeh et al. 2006). In addition, the latter motor bias should have led to the same peak of left premotor activation in both OBTtasks. We propose that this right hemispheric lateralization in premotor cortex might be due to self processing (Keenan et al. 2000) that is involved in both OBT-tasks as participants were asked to mentally imagine themselves at the indicated position and orientation (Blanke et al. 2005; see also below). We argued that the present type of mental own body imagery is functionally linked to self processing with respect to visuo-spatial perspective taking and self location in the right hemisphere processing at the TPJ (Blanke and Arzy 2005; Blanke and Mohr 2005) as well as right premotor and prefrontal cortex (Keenan et al. 2000; Decety and Sommerville 2003; Gillihan and Farah 2005).

\section{Extrastriate Cortex}

Two areas in extrastriate cortex were found to show stimulus selectivity, FFA and EBA. Right FFA was activated during the presentation of full and upper bodies and showed strongest activation for the latter stimuli, independently of the task. Left EBA was significantly activated only during the presentation of full bodies and this activation was dependent on mental imagery suggesting that FFA and EBA are implicated differently in mental own body transformations.

Concordant with the predominant role of the FFA in face perception (Kanwisher et al. 1997; McCarthy et al. 1997) activation in this area was observed independent of task and predominated for upper body stimuli that contained large faces: FFA was activated during mental imagery of faces, but also during mental imagery of the full body, with a stronger activity during the former than the latter. The predominance of right hemispheric FFA activation has been reported in previous neuroimaging studies (Kanwisher et al. 1997; McCarthy et al. 1997) and is consistent with the clinical finding that prosopagnosia may follow bilateral or right hemispheric damage to fusiform and lingual gyrus including the FFA (Landis et al. 1998; Rossion et al. 2003).

Whereas previous studies investigated the role of the EBA in the perception of human bodies (Downing et al. 2001; Grossman and Blake 2002; Urgesi et al. 2007), our data show that the EBA is also involved in mental imagery of human bodies. Supporting previous notions that the EBA activation is stronger when presented with full human bodies, the present EBA activation was not observed for mental imagery of upper bodies (Downing et al. 2001; Grossman and Blake 2002; Astafiev et al. 2004). We cannot exclude that this was partly due to the presence of a large face in the stimuli depicting upper bodies. Yet, faces were also present in the full body stimuli as well as the lateralization task. Moreover, in half of the trials the schematic human figures were depicted from the back without faces suggesting that the EBA is primarily involved in mental imagery of full human bodies.

We observed this activation only for the left EBA. Although the lateralization of EBA's activity in response to visual bodily stimuli is still an open debate, no lateralization was observed in some previous fMRI work (Grossman and Blake 2002; Saxe and Kanwisher 2003; Astafiev et al. 2004; Hodzic et al. 2009a), whereas a differential EBA lateralization was observed when bodily identity and action discrimination were tested (Hodzic et al. 2009b; Urgesi et al. 2007). Other fMRI studies found greater consistency (Downing et al. 2001) and selectivity (Downing et al. 2006a; Downing et al. 2006b) for the right EBA or a general greater whole-body selectivity in the right versus left EBA (Downing et al. 2007; Aleong and Paus 2010; Proverbio et al. 2008). Our results at the level of the EBA are in agreement with results of a previous study that also investigated mental imagery of full human body stimuli (Zacks et al. 1999) and reported bilateral activations near the EBA with a left hemisphere predominance. More recently, we have found that the left EBA during mental imagery of full bodies was more strongly recruited using electrical neuroimaging (Arzy et al. 2006).

Finally it might be argued that the stronger activation in left EBA during the OBTf task with respect to the LATf task, could be the result of the attention directed to visually presented bodies, that is the stimulus EBA is mostly selective to. Yet, we observed an activation of left EBA, which has been reported to be involved in the imagery of human bodies, whereas the right EBA is more commonly involved in the visual perception of human bodies (Downing et al. 2007; Aleong and Paus 2010; Proverbio et al. 2008). Thus, if the attention would have interfered with the present EBA activation, it should have rather influenced right EBA, which is not the case.

Based on the present and previous data we speculate that visual perception of human bodies might be associated 
with bilateral EBA activation (Downing et al. 2001; Grossman and Blake 2002; Saxe and Kanwisher 2003; Astafiev et al. 2004) with a small predominance of the right EBA, whereas imagery of full human bodies might preferentially activate left EBA (Zacks et al. 1999; Arzy et al. 2006; present study).

\section{Own Body Imagery, Self, and the Right Hemisphere}

We found that mental imagery of full and upper bodies activated a large, mainly right hemisphere network consisting of TPJ, premotor cortex and bilateral parietal cortex (with a right predominance). Because a right hemisphere dominance was found in the present study for partial upper bodies as well as full bodies, we suggest that the latter network of brain regions as well as their right hemisphere lateralization overlaps with regions that have been shown to be activated during self-related processing (Farrer and Frith 2002; Farrer et al. 2003; Chaminade and Decety 2002; Ruby and Decety 2001, 2003, 2004). Based on this we suggest that when people are asked to imagine themselves at the position of a depicted schematic body, mental imagery is likely to rely on distributed right hemispheric neural mechanisms involved in self processing, largely independent of whether a full or upper body is shown. This localization and lateralization of brain activation with respect to upper and full bodies has also been found following electrical cortical stimulation (Blanke et al. 2002; De Ridder et al. 2007) and is concordant with clinical data from patients with illusory own body perceptions such as out-of-body experiences and autoscopic hallucinations (Blanke and Mohr 2005). Thus, during out-of-body experiences patients generally perceive a full human body and out-of-body experiences have been linked to the right TPJ (Blanke et al. 2005; Blanke and Mohr 2005). This differs from the body perceived during autoscopic hallucinations, when patients mostly describe seeing a partial upper body. While patients with autoscopic hallucinations mostly suffer from brain damage or interference in extrastriate cortex, patients with out-of-body experiences suffer from right hemispheric lesions, especially in temporo-parietal cortex (Blanke et al. 2004; Blanke and Mohr 2005; Devinsky et al. 1989). These phenomenological differences reinforced by recent neuroimaging data showing that faces (i.e., upper bodies) are processed in at least partly distinct brain areas, such as the fusiform and occipital face areas (Kanwisher et al. 1997; Puce et al. 1998), whereas full bodies are processed in the fusiform and extrastriate body area (Arzy et al. 2006, 2007; Blanke et al. 2005; Downing et al. 2001; Zacks et al. 1999, 2002). However, further work is needed to clarify this asymmetric pattern of activation for faces, partial bodies, and full bodies.
The spatial location of an object can be represented at least with respect to two different frames of reference: egocentric and allocentric (see, e.g., Howard and Templeton 1964; Lacquaniti et al. 1997). In an egocentric frames the spatial coordinates of an object are encoded with respect to the observer's body. While, in an allocentric frames the spatial coordinates of an object are represented on the base of external objects (Kosslyn 1994; Galati et al. 2000; Vogeley and Fink 2003; Jordan et al. 2004; Zaehle et al. 2007). Classically the OBT-task is performed using an egocentric frame of reference, while the LAT-task is a control task not asking for a transformation with respect to either the egocentric nor the allocentric frame of reference. Previous research, and in particular the direct comparison between the mental imagery of one's own versus another person's full body (Corradi-Dell'Acqua et al. 2009) has shown that both hemispheres are differentially involved in processing one's own as opposed to other's body (e.g., Ruby and Decety 2001; Vogeley and Fink 2003; Decety and Sommerville 2003). We note that the main interest of the present fMRI study-motivated by insights from data of neurological patients with illusory own body perceptions (Blanke et al. 2004; Blanke and Mohr 2005)—was to distinguish mechanisms of mental imagery concerning the full body from those of the upper body with respect to the LAT control task. Here we did not control for the use of egocentric versus allocentric mechanisms (as done by i.e., Arzy et al. 2006) and others (Kosslyn 1994; Galati et al. 2000; Vogeley and Fink 2003) and can accordingly not exclude that our results are partly affected by such differential influences.

It has been proposed that motor and visual imagery can engage different brain networks (Sirigu and Duhamel 2001; Tomasino and Rumiati 2004), that kinaesthetic/motor imagery, but not visual imagery, is effective in the activation of specific motor pathways (Fourkas et al. 2006) and that vestibular mechanisms may also play an important role in imagery (Grabherr et al. 2007; Mast et al. 2006; Mast and Meissner 2004; Blanke et al. 2004). However the present study was not planned to directly investigate the contributions of motor, visual and vestibular mechanisms in mental imagery. Thus at present we cannot affirm or exclude whether the performance of our participants during mental imagery relied more on mechanisms related to visual imagery, motor imagery or vestibular imagery and how this may have influenced the observed brain activations.

Acknowledgements The authors would like to thank Shahar Arzy and Istvan Molnar-Szakacs for their helpful comments on a previous version of the manuscript. This work was supported by the Leenaards Foundation, the Swiss National Science Foundation (Grants 3100067874.02) and the Fondation de Famille Sandoz. This work was also supported by the Centre d'Imagerie BioMédicale (CIBM) of the 
University of Lausanne, the Swiss Federal Institute of Technology Lausanne, the University of Geneva, the Centre Hospitalier Universitaire Vaudois (CHUV), the Hôpitaux Universitaires de Genève (HUG), and the Jeantet Foundation.

\section{References}

Aleong R, Paus T (2010) Neural correlates of human body perception. J Cogn Neurosci 22(3):482-495

Alivisatos B, Petrides M (1997) Functional activation of the human brain during mental rotation. Neuropsychologia 35:111-118

Allison T, Puce A, McCarthy G (2000) Social perception from visual cues. Trends Cogn Sci 4:267-278

Arzy S, Thut G, Mohr C, Michel CM, Blanke O (2006) Neural basis of embodiment: distinct contributions of temporoparietal junction and extrastriate body area. J Neurosci 26:8074-8081

Arzy S, Mohr C, Michel CM, Blanke O (2007) Duration and not strength of activation in temporo-parietal cortex positively correlates with schizotypy. Neuroimage 35(1):326-333

Astafiev SV, Stanley CM, Shulman GL, Corbetta M (2004) Extrastriate body area in human occipital cortex responds to the performance of motor actions. Nat Neurosci 7:542-548

Aziz-Zadeh L, Iacoboni M, Zaidel E (2006) Hemispheric sensitivity to body stimuli in simple reaction time. Exp Brain Res 170: 116-121

Berlucchi G, Aglioti S (1997) The body in the brain: neural bases of corporeal awareness. Trends Neurosci 20(12):560-564

Blanke O, Arzy S (2005) The out-of-body experience: disturbed selfprocessing at the temporo-parietal junction. Neuroscientist 11(1):16-24

Blanke O, Metzinger T (2009) Full-body illusions and minimal phenomenal selfhood. Trends Cogn Sci 13(1):7-13

Blanke O, Mohr C (2005) Autoscopic phenomena of neurological origin. Implications for corporal awareness and self consciousness. Brain Res Rev 50:184-199

Blanke O, Ortigue S, Landis T, Seeck M (2002) Inducing illusory own-body perceptions. Nature 419:269-270

Blanke O, Landis T, Spinelli L, Seeck M (2004) Out-of-body experience and autoscopy of neurological origin. Brain 127: 243-258

Blanke O, Mohr C, Michel CM, Pascual-Leone A, Brugger P, Seeck M, Landis T, Thut G (2005) Linking OBEs and self processing to mental own body imagery at the temporo-parietal junction. J Neurosci 25:550-557

Bonda E, Petrides M, Frey S, Evans M (1995) Neural correlates of mental transformations of the body-in-space. Proc Natl Acad Sci 92:11180-11184

Brugger P (2002) Reflective mirrors: perspective-taking in autoscopic phenomena. Cogn Neuropsychiatr 7:179-194

Burgess N (2008) Spatial cognition and the brain. Ann N Y Acad Sci 1124:77-97

Carpenter PA, Just MA, Keller TA, Eddy W, Thulborn K (1999) Graded functional activation in the visuospatial system with the amount of task demand. J Cogn Neurosci 11:9-24

Chaminade T, Decety J (2002) Leader or follower? Involvement of the inferior parietal lobule in agency. Neuroreport 13(15):19751978

Cohen YE, Andersen RA (2002) A common reference frame for movement plans in the posterior parietal cortex. Nat Rev Neurosci 3(7):553-562

Colby CL, Duhamel JR (1996) Spatial representations for action in parietal cortex. Brain Res Cogn Brain Res 5(1-2):105-115

Corradi-Dell' Acqua C, Tomasino B, Fink GR (2009) What is the position of an arm relative to the body? Neural correlates of body schema and body structural description. J Neurosci 29(13):41624171

Creem SH, Downs TH, Wraga M, Harrington GS, Proffitt DR, Downs JH (2001) An fMRI study of imagined self-rotation. Cogn Affect Behav Neurosci 1:239-249

de Jong BM, van der Graaf FH, Paans AM (2001) Brain activation related to the representations of external space and body scheme in visuomotor control. Neuroimage 14(5):1128-1135

De Ridder D, Van Laere K, Dupont P, Menovsky T, Van de Heyning $\mathrm{P}$ (2007) Visualizing out-of-body experience in the brain. N Engl J Med 357(18):1829-1833

Decety J, Sommerville JA (2003) Shared representations between self and other: a social cognitive neuroscience view. Trends Cogn Sci 7:527-533

Devinsky O, Feldmann E, Burrowes K, Bromfield E (1989) Autoscopic phenomena with seizures. Arch Neurol 46:1080-1088

Downing PE, Jiang Y, Shuman M, Kanwisher N (2001) A cortical area selective for visual processing of the human body. Science 293:2470-2473

Downing PE, Chan AW, Peelen MV, Dodds CM, Kanwisher N (2006a) Domain specificity in visual cortex. Cereb Cortex 16:1453-1461

Downing PE, Peelen MV, Wiggett AJ, Tew BD (2006b) The role of the extrastriate body area in action perception. Soc Neurosci 1:52-62

Downing PE, Wiggett AJ, Peelen MV (2007) Functional magnetic resonance imaging investigation of overlapping lateral occipitotemporal activations using multi-voxel pattern analysis. J Neurosci 27:226-233

Farrer C, Frith CD (2002) Experiencing oneself vs. another person as being the cause of an action: the neural correlates of the experience of agency. Neuroimage 15(3):596-603

Farrer C, Franck N, Georgieff N, Frith CD, Decety J, Jeannerod M (2003) Modulating the experience of agency: a positron emission tomography study. Neuroimage 18(2):324-333

Fasold O, von Brevern M, Kuhberg M, Ploner CJ, Villringer A, Lempert T, Wenzel R (2002) Human vestibular cortex as identified with caloric stimulation in functional magnetic resonance imaging. Neuroimage 17(3):1384-1393

Fourkas AD, Ionta S, Aglioti SM (2006) Influence of imagined posture and imagery modality on corticospinal excitability. Behav Brain Res 168(2):190-196

Friston KJ, Worsley KJ, Frackowiak RSJ, Mazziotta JC, Evans AC (2003) Assessing the significance of focal activations using their spatial extent. Hum Brain Mapp 1:210-220

Galati G, Lobel E, Vallar G, Berthoz A, Pizzamiglio L, Le Bihan D (2000) The neural basis of egocentric and allocentric coding of space in humans: a functional magnetic resonance study. Exp Brain Res 133(2):156-164

Gillihan JS, Farah JM (2005) Is the self special? A critical review of evidence from experimental psychology and cognitive neuroscience. Psychol Bull 131:75-97

Grabherr L, Bach S, Indermaur K, Metzler S, Mast F (2007) Mental transformations of bodies and body-parts in microgravity. J Vest Res 17(5-6):279-287

Grossman ED, Blake R (2002) Brain areas active during visual perception of biological motion. Neuron 35:1167-1175

Guariglia C, Piccardi L, Puglisi Allegra MC, Traballesi M (2002) Is autotopoagnosia real? EC says yes. A case study. Neuropsychologia 40:1744-1749

Harris IM, Miniussi C (2003) Parietal lobe contribution to mental rotation demonstrated with rTMS. J Cogn Neurosci 15:315-323

Henson RN, Rylands A, Ross E, Vuilleumeir P, Rugg MD (2004) The effect of repetition lag on electrophysiological and haemodynamic correlates of visual object priming. Neuroimage 21(4):1674-1689 
Hodzic A, Kaas A, Muckli L, Stirn A, Singer W (2009a) Distinct cortical networks for the detection and identification of human body. NeuroImage 45(4):1264-1271

Hodzic A, Muckli L, Singer W, Stirn A (2009b) Cortical responses to self and others. Hum Brain Mapp 30(3):951-962

Howard IP, Templeton WB (1964) Visually-induced eye torsion and tilt adaptation. Vision Res 4(7):433-437

Howard RR, Brammer M, Wright I, Woodruff PW, Bullmore ET, Zeki S (1996) A direct demonstration of functional specialisation within motion-related visual and auditory cortex of the human brain. Curr Biol 6(8):1015-1019

Ionta S, Fourkas A, Aglioti SM (2010) Egocentric and object-based transformations in the laterality judgement of human and animal faces and of non-corporeal objects. Behav Brain Res 207(2): $452-457$

Jordan K, Schadow J, Wuestenberg T, Heinze HJ, Jancke L (2004) Different cortical activations for subjects using allocentric or egocentric strategies in a virtual navigation task. Neuroreport 15(1):135-140

Kanwisher N, McDermott J, Chun M (1997) The fusiform face area: a module in human extrastriate cortex specialized for face perception. J Neurosci 17:4302-4311

Kawamichi H, Kikuchi Y, Noriuchi M, Senoo A, Ueno S (2007) Distinct neural correlates underlying two- and three-dimensional mental rotations using three-dimensional objects. Brain Res 1144:117-126

Keenan JP, McCutcheon B, Freund S, Gallup GG Jr, Sanders G, Pascual-Leone A (1999) Left hand advantage in a self-face recognition task. Neuropsychologia 37(12):1421-1425

Keenan JP, Wheeler MA, Gordon GG, Pascual-Leone A (2000) Self recognition and the right prefrontal cortex. Trends Cogn Sci $4: 338-344$

Klöppel S, Vongerichten A, van Eimeren T, Frackowiak RS, Siebner HR (2007) Can left-handedness be switched? Insights from an early switch of handwriting. J Neurosci 27(29):7847-7853

Knyazeva MG, Fornari E, Meuli R, Innocenti G, Maeder P (2006) Imaging of a synchronous neuronal assembly in the human visual brain. Neuroimage 9(2):593-604

Kosslyn SM (1994) Image and brain: the resolution of the imagery debate. MIT Press, Cambridge, MA

Kosslyn SM, Digirolamo GJ, Thompson WL, Alpert NM (1998) Mental rotation of objects versus hands: neural mechanisms revealed by positron emission tomography. Psychophysiol 35:151-161

Lacquaniti F, Perani D, Guigon E, Bettinardi V, Carrozzo M, Grassi F, Rossetti Y, Fazio F (1997) Visuomotor transformations for reaching to memorized targets: a PET study. Neuroimage 5(2):129-146

Landis T, Regard M, Bliestle A, Kleihues P (1998) Prosopagnosia and agnosia for noncanonical views. An autopsied case. Brain 111:1287-1297

Lenggenhager B, Smith ST, Blanke O (2006) Functional and neural mechanisms of embodiment: importance of the vestibular system and the temporal parietal junction. Rev Neurosci 17(6):643-657

Leube DT, Knoblich G, Erb M, Grodd W, Bartels M, Kircher TTJ (2003) The neural correlates of perceiving one's own movements. NeuroImage 20:2084-2090

Lobel E, Kleine JF, Leroy-Willig A, Van de Moortele PF, Le Bihan D, Grüsser OJ, Berthoz A (1999) Cortical areas activated by bilateral galvanic vestibular stimulation. Ann N Y Acad Sci 871:313-323

Maeder PP, Meuli RA, Adriani M, Bellmann A, Fornari E, Thiran JP, Pittet A, Clarke S (2001) Distinct pathways involved in sound recognition and localization: a human fMRI study. Neuroimage 14(4):802-816
Maguire EA, Burgess N, Donnett JG, Frackowiak RS, Frith CD, O'Keefe J (1998) Knowing where and getting there: a human navigation network. Science 280:921-924

Mast FW, Meissner F (2004) Mental transformations of perspective during whole-body roll-rotation. J Vestib Res 14:113

Mast FW, Merfeld DM, Kosslyn SM (2006) Visual mental imagery during caloric vestibular stimulation. Neuropsychologia 44: 101-109

McCarthy G, Puce A, Gore JC, Allison T (1997) Face-specific processing in the human fusiform gyrus. J Cogn Neurosci 9: $605-610$

Mohr C, Blanke O, Brugger P (2006) Perceptual aberrations impair mental own body transformations. Behav Neurosci 120:528-534

Ogden JA (1985) Autotopagnosia. Occurrence in a patient without nominal aphasia and with an intact ability to point to parts of animals and objects. Brain 108:1009-1022

Oldfield RC (1971) The assessment and analysis of handedness: the Edinburgh Inventory. Neuropsychologia 9:97-113

Overney LS, Blanke O (2008) Impaired Imagery for Upper Limbs. Brain Topogr 22(1):27-43

Overney LS, Michel CM, Harris IM, Pegna AJ (2005) Cerebral processes in mental transformations of body parts: recognition prior to rotation. Cogn Brain Res 25:722-734

Parsons LM (1987a) Imagined spatial transformation of one's body. J Exp Psychol Gen 116:172-191

Parsons LM (1987b) Imagined spatial transformations of one's hands and feet. Cogn Psychol 19:178-241

Parsons LM, Fox PT, Downs JH, Glass T, Hirsch TB, Martin CC, Jerabek PA, Lancaster JL (1995) Use of implicit motor imagery for visual shape discrimination as revealed by PET. Nature 375:54-58

Pegna AJ, Khateb A, Spinelli L, Seeck M, Landis T, Michel CM (1997) Unraveling the cerebral dynamics of mental imagery. Hum Brain Map 5:410-421

Proverbio AM, Zani A, Adorni R (2008) Neural markers of a greater female responsiveness to social stimuli. BMC Neurosci 9:56

Puce A, Allison T, Bentin S, Gore JC, McCarthy G (1998) Temporal cortex activation in humans viewing eye and mouth movements. J Neurosci 18:2188-2199

Reed CL, Stone DE, Grubb JD, McGoldrick JD (2006) Turning configural processing upside down. Part and whole body postures. J Exp Psychol 32:37-87

Rossion B, Caldara R, Seghier M, Schuller MA, Lazeyras F, Mayer E (2003) A network of occipito-temporal face-sensitive areas besides the right middle fusiform gyrus is necessary for normal face processing. Brain 126:2381-2395

Ruby P, Decety J (2001) Effect of subjective perspective taking during simulation of action: a PET investigation of agency. Nat Neurosci 4:546-550

Ruby P, Decety J (2003) What you believe versus what you think they believe: a neuroimaging study of conceptual perspective-taking. Eur J Neurosci 17(11):2475-2480

Ruby P, Decety J (2004) How would you feel versus how do you think she would feel? A neuroimaging study of perspectivetaking with social emotions. J Cogn Neurosci 16(6):988-999

Saxe R, Kanwisher N (2003) People thinking about thinking people. The role of the temporo-partietal junction in theory of mind. NeuroImage 19:1835-1842

Schwoebel J, Coslett HB (2005) Evidence for multiple, distinct representations of the human body. J Cogn Neurosci 17:543-553

Sirigu A, Duhamel JR (2001) Motor and visual imagery as two complementary but neurally dissociable mental processes. J Cogn Neurosci 13:910-919

Sirigu A, Grafman J, Bressler K, Sunderland T (1991) Multiple representations contribute to body knowledge processing. Evidence from a case of autotopagnosia. Brain 114:629-642 
Tomasino B, Rumiati RI (2004) Effects of strategies on mental rotation and hemispheric lateralization: neuropsychological evidence. J Cogn Neurosci 16(5):878-888

Urgesi C, Candidi M, Ionta S, Aglioti SM (2007) Representation of body identity and body actions in extrastriate body area and ventral premotor cortex. Nat Neurosci 10(1):30-31

Vaina LM, Solomon J, Chowdhury S, Sinha P, Belliveau JW (2001) Functional neuroanatomy of biological motion perception in humans. Proc Natl Acad Sci USA 98:11656-11661

Vallar G, Lobel E, Galati G, Berthoz A, Pizzamiglio L, Le Bihan D (1999) A fronto-parietal system for computing the egocentric spatial frame of reference in humans. Exp Brain Res 124:281-286

Vingerhoets G, Santens P, Van Laere K, Lahorte P, Dierchx RA, De Reuck J (2001) Regional brain activity during different paradigms of mental rotation in healthy volunteers: a positron emission tomography study. Neuroimage 13:381-391

Vogeley K, Fink GR (2003) Neural correlates of the first-personperspective. Trends Cogn Sci 7(1):38-42

Volcic R, Kappers AM (2008) Allocentric and egocentric reference frames in the processing of three-dimensional haptic space. Exp Brain Res 188(2):199-213
Wraga M, Creem SH, Proffitt DR (1999) The influence of spatial reference frames on imagined object- and viewer rotations. Acta Psychol 102:247-264

Wraga M, Shephard JM, Church JA, Inati S, Kosslyn SM (2005) Imagined rotations of self versus objects: an fMRI study. Neuropsychologia 43(9):1351-1361

Zacks JM, Rypma B, Gabriel J, Tversky B, Glover G (1999) Imagined transformations of bodies: an fMRI investigation. Neuropsychologia 37:1029-1040

Zacks JM, Ollinger JM, Sherdian MA, Tversky B (2002) A parametric study of mental spatial transformations of bodies. NeuroImage 16:857-872

Zacks JM, Vettel JM, Michelon P (2003) Imagined viewer and object rotations dissociated with event-related FMRI. J Cogn Neurosci 15(7):1002-1018

Zaehle T, Jordan K, Wüstenberg T, Baudewig J, Dechent P, Mast FW (2007) The neural basis of the egocentric and allocentric spatial frame of reference. Brain Res 1137(1):92-103 